\title{
Primeira Ajuda em Saúde Mental: Contributo do programa para o incremento da literacia em saúde mental dos estudantes do ensino superior
}

\author{
Mental Health First Aid: Contribution of the program to increase mental \\ health literacy in higher education students
}

\author{
Luís Manuel Jesus Loureiro (1) Amorim Gabriel Santos Rosa (1) Carla Marina Frajuca Pimentel (2) \\ Sandrina Ribeiro da Cunha (3) Lúcia Valente da Costa (4) Susana Isabel Pereira Correia (2) Tânia \\ Manuela Moço Morgado (5) Ângela Dionísio Marques (1) Daniela Maria Miranda Santos (6) \\ Mariana João Jorge Albino (7)

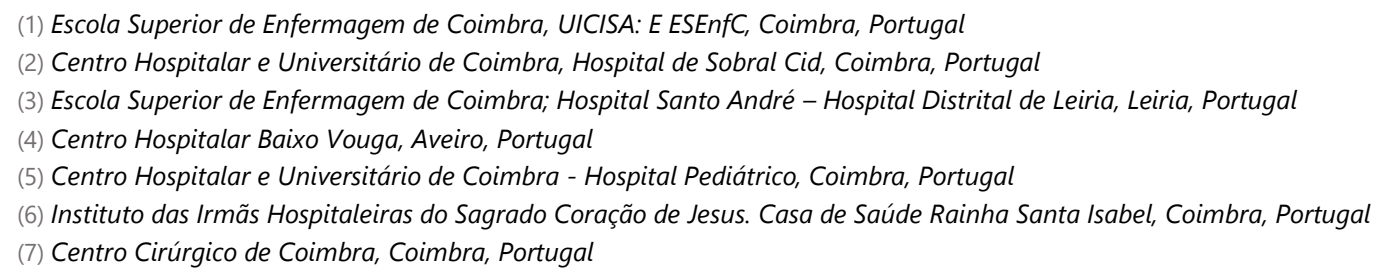

Recebido: 21/07/2020; Revisto: 20/10/2020; Aceite: 02/11/2020.

\begin{abstract}
Resumo
Objetivo: O presente estudo tem como objetivo avaliar o contributo em termos da efetividade do programa de Primeira Ajuda em Saúde Mental na literacia em saúde mental acerca da depressão, a partir de uma amostra de estudantes de Enfermagem aquando do ingresso e integração ao curso. Métodos: Utilizou-se um desenho pré-experimental com grupo único, avaliação pré e pós intervenção. O Programa teve a duração de um dia (9 horas). A amostra do estudo foi constituída por 100 estudantes do $1^{\circ}$ ano do curso de Enfermagem (na integração ao curso), tendo sido selecionados de modo aleatório simples com recurso ao software random.org. A média das idades foi de 18,54 anos ( $D P=2,00$ anos). Como instrumentos de colheita de dados foram utilizados o Questionário de Avaliação da Literacia em Saúde Mental, aplicado à depressão, a versão breve do Inventário de Crenças acerca das Doenças Mentais e a Escala de Avaliação do Estigma Pessoal. Recorreu-se às estatísticas resumo, aos testes de McNemar e $t$ de Student para grupos emparelhados e, como medidas de tamanho de efeito, o $g$ e o $d$ respetivamente. Resultados: Observou-se com a intervenção um incremento da literacia em saúde mental ao nível do reconhecimento da depressão e estratégias comunicacionais de prestação de primeira ajuda $(p<0,05)$, especificamente na adequação e utilidade de valorizar sintomas e não expressar julgamentos, assim como uma redução das atitudes estigmatizantes acerca das doenças e doentes $(p<0,05)$. Conclusões: Apesar das limitações relacionadas com o desenho utilizado, nomeadamente a não existência de grupo de controlo, os resultados indicam que a frequência do programa contribui para aumentar a literacia em saúde mental e reduzir o estigma associado aos problemas de saúde mental.
\end{abstract}

Palavras-Chave: Primeira ajuda; Literacia em saúde mental; Depressão; Ensino superior; Quase-experiência.

\section{DI\&D | ISMT}

rpics@ismt.pt https://rpics.ismt.pt

\section{Publicação em Acesso Aberto}

(c) 2020. O(s) Autor(es). Este é um artigo de acesso aberto distribuído sob a Licença Creative Commons Attribution, que permite uso, distribuição e reprodução sem restrições em qualquer meio, desde que o trabalho original seja devidamente citado.

\section{Luís Loureiro}

Avenida Bissaya Barreto Apartado 7001

$3046-851$, Coimbra, Portugal

Tel.: 239. 802850

E-mail: luisloureiro@eenfc.pt 


\begin{abstract}
Objective: The present study aims to evaluate the contribution in terms of the Mental Health First Aid program's effectiveness in mental health literacy about depression, based on a sample of nursing students when entering and integrating into the course. Method: A pre-experimental design with a single group design, pre- and post-intervention assessment was used. The program lasted one day ( 9 hours). The study sample consisted of 100 students from the $1 \mathrm{st}$ year of the Nursing course (in the integration to the course), having been selected in a simple random way using the software random.org. The average age was 18.54 years $(S D=2.00$ years). For data collection, we used the Mental Health Literacy Assessment Questionnaire, applied to depression, the short version of the Beliefs about Mental Illnesses Inventory, and the Personal Stigma Assessment Scale. We calculated summary statistics, McNemar and Student's $t$-tests for paired groups, and measures of effect size, the $g$, and $d$, respectively. Results: An increase in mental health literacy was observed with the recognition of depression and communication strategies for providing first aid $(p<.05)$, specifically in the adequacy and usefulness of valuing symptoms and not expressing judgments, as well as a reduction in stigmatizing attitudes about disorders and patients $(p<0.05)$. Conclusions: Despite the limitations related to the design used, namely the lack of a control group, the results indicate that the frequency of the program contributes to increase mental health literacy about depression and reduce the stigma associated with mental health problems.
\end{abstract}

Keywords: First aid; Mental health literacy; Depression, Higher education; Quasi-experience.

\title{
Introdução
}

A expressão Primeira Ajuda em Saúde Mental (PASM) foi utilizada no contexto Português de forma pioneira, consistente e estruturada em 2010, a partir dos trabalhos de uma equipa de investigadores da Unidade de Investigação em Ciências da Saúde: Enfermagem da Escola Superior de Enfermagem de Coimbra (Loureiro et al., 2012), no âmbito de uma intervenção de educação e sensibilização para a saúde mental de adolescentes e jovens. A intervenção assentou na criação de raiz de uma plataforma web (https://felizmente.esenfc.pt/felizmente/), sendo a expressão utilizada para nomear um dos separadores da plataforma ("Primeira Ajuda em Saúde Mental - Ajudar um Amigo"), englobando, entre outros, o algoritmo ANIPI (Loureiro et al., 2014), resultado da tradução e adaptação do algoritmo ALGEE do programa original australiano designado por Mental Health First Aid (MHFA ${ }^{\circledR}$ ) (Kitchener \& Jorm, 2002; Loureiro et al., 2014). O que se pretendia com a informação constante no separador era ensinar as estratégias e ações para prestar PASM, ensinando adolescentes e jovens a prestar ajuda e apoio a um amigo ou colega, que estivesse a desenvolver um problema relacionado com a sua saúde mental no sentido de favorecer a procura de ajuda, e abrangia diferentes cenários/situações de adoecer mental (Loureiro \& Sousa, 2019).

Ainda na atualidade a mnemónica ANIPI significa (Loureiro, 2014, p. 23):
A - Aproximar-se da pessoa, observar e ajudar (incluindo situações de crise);
N - Não julgar e escutar com atenção;
I - Informar e apoiar;
P - Procurar ajuda profissional especializada incentivando a pessoa a obtê-la;
I - Incentivar o recurso a outros apoios. 
Posteriormente a expressão veio a dar título ao livro Primeira Ajuda em Saúde Mental de Loureiro (2014) cujo conteúdo comtempla as linhas orientadoras e conteúdos do designado programa, em linha com o programa internacional MHFA ${ }^{\circledR}$ (Kitchener \& Jorm, 2002).

Este trabalho, desenvolvido em torno do programa e da plataforma web, teve como génese o estudo acerca das representações sociais das doenças mentais e sua importância para a promoção da saúde mental (Loureiro, 2008; Loureiro et al., 2008), surgindo ancorado ao conceito de Literacia em Saúde Mental (LSM) (Jorm et al., 1997) e ao programa MHFA ${ }^{\circledR}$ (Kitchener \& Jorm, 2002).

Hodiernamente, o programa de PASM ${ }^{\circledR}$ contempla uma estrutura modular cujos conteúdos se centram na educação e formação em termos de todas as componentes da literacia em saúde mental (Jorm, 2019), destacando-se o ensino da prestação de primeira ajuda interpares, apoio e suporte a alguém que esteja a sofrer de um problema relacionado com a sua saúde mental, incluindo, na linha do programa MHFA ${ }^{\circledR}$, o ensino de primeiros socorros situações de crise em saúde mental como são exemplo os ataques de pânico, ideação suicida, entre outras situações.

Neste sentido a expressão PASM engloba, quer o nome do programa no contexto português (PASM ${ }^{\circledR}$ ), quer ainda a ação que constitui um dos focos centrais do programa e que deriva da componente da LSM designada de conhecimentos e competências para prestar primeira ajuda e primeiros socorros aos outros Jorm, 2019). A ação de primeira ajuda compreende, entre outros aspetos, a preparação da abordagem à pessoa, a aproximação e escolha do momento e local adequados, a valorização dos sintomas e não expressão de julgamentos; a expressão de preocupação e disponibilidade para ajudar com enfâse para as atitudes comunicacionais, assim como o compromisso de ajuda (Loureiro, 2020).

Ainda que em termos de estrutura o programa siga as linhas orientadoras do programa MHFA ${ }^{\circledR}$, nomeadamente na conservação de uma estrutura baseada em módulos e com carga horária total entre as nove e as 14 horas; no programa de PASM foram introduzidos conteúdos relativos a todas as componentes da LSM, incluindo uma abordagem a outros problemas de saúde mental (e.g., ansiedade às situações de exame/avaliação), o ensino de estratégias de autoajuda que incluem por exemplo técnicas de relaxamento, assim como conteúdos baseados na melhor evidência relativos à prevenção dos problemas de saúde mental no quotidiano (e.g., alimentação, higiene do sono, exercício).

No contexto do ensino superior português, o programa de PASM tem sido aplicado especificamente a estudantes de Enfermagem, sendo alvo de diferentes avaliações de efetividade (Cunha, 2019; Loureiro \& Costa, 2019; Loureiro \& Freitas, 2020; Loureiro \& Sousa; 2019). A evidência produzida neste contexto, a exceção do estudo de Loureiro e Sousa (2019) que reporta aos resultados do estudo piloto, revelam que o programa contribui para o incremento da LSM em termos de reconhecimentos dos problemas (depressão/ansiedade), intenção de procura de ajuda em saúde mental e confiança para prestar primeira ajuda. As medidas de tamanho efeito utilizadas para comparar os resultados antes e depois das intervenções nos estudos de Loureiro e Costa (2019), Cunha (2019) e Loureiro e Freitas (2020), apontam para valores de moderados a elevados para todas as dimensões referidas. É de salientar que todos os 
estudos seguem desenhos pré-experimentais com grupo único, avaliação pré e pós intervenção, utilizando o QuALiSMental (Loureiro, 2015) como instrumento de medida, sendo estudadas amostras de estudantes de Enfermagem, com tamanhos que variam de $n=36$ (Cunha, 2019) a $n=219$ (Loureiro \& Freitas, 2020). No contexto internacional os estudos realizados em ensino superior, especificadamente de Enfermagem, são muito reduzidos, conforme referido por Loureiro e Freitas (2020).

Este estudo tem, assim, como objetivo avaliar o contributo em termos de efetividade do programa de PASM na literacia em saúde mental acerca da depressão, a partir de uma amostra de estudantes de Enfermagem aquando do ingresso e integração no curso de licenciatura.

\section{Método}

O presente estudo teve natureza quantitativa, prosseguindo um desenho pré-experimental com grupo único, avaliação pré e pós intervenção.

\section{Hipótese de investigação}

O programa de PASM ${ }^{\circledR}$ contribui para o incremento da LSM dos estudantes do $1 .^{\circ}$ ano do curso de Enfermagem nas componentes de reconhecimento da depressão, conhecimentos acerca da prestação de primeira ajuda e redução das crenças e atitudes estigmatizantes associadas.

\section{Participantes}

A amostra foi constituída por 100 estudantes do primeiro ano do curso de Enfermagem, que foram submetidos ao programa de PASM, sendo a maioria dos participantes do sexo feminino (88,00\%). A média das idades foi de 18,54 anos ( $D P=2,00$ anos), sendo a idade mínima observada de 17 anos e máxima de 30 anos.

O coeficiente de variação foi de 10,8\% o que mostra que o grupo era homogéneo em termos da variável idade. A rácio de variação da variável sexo indicou um valor de 0,12 , sugestivo de baixa dispersão, valor que é aceitável face à natureza do curso de enfermagem, maioritariamente frequentado por estudantes do sexo feminino.

\section{Instrumentos}

\section{Questionário de Avaliação da Literacia em Saúde Mental (QuaLiSMental)}

O QuaLiSMental (Loureiro, 2015), na versão reduzida para a depressão, inclui as questões para a componente de reconhecimento dos problemas e perturbações mentais de modo a promover e facilitar a procura de ajuda e uma grelha de expressões utilizadas no quotidiano na abordagem à pessoa que está em sofrimento ou a desenvolver um problema de saúde mental. O QuALiSmental apresenta validade de 
construto e bons índices de consistência interna tal como referido no estudo psicométrico do instrumento (Loureiro, 2015).

O questionário é precedido por uma vinheta que relata um caso de depressão, de acordo com os critérios de diagnóstico da DSM-5 (Associação Americana de Psiquiatria, 2014), tal como se apresenta de seguida:

A Sofia é uma jovem de 18 anos que se tem sentido invulgarmente triste durante as últimas semanas. Sente-se sempre cansada e tem problemas para adormecer e manter o sono. Perdeu o apetite e ultimamente tem vindo a perder peso. Tem dificuldade em concentrar-se no estudo e as suas notas desceram. Mesmo as tarefas do dia-a-dia the parecem muito difíceis, pelo que tem adiado algumas decisões. Os seus pais e amigos estão muito preocupados com ela.

\section{Inventário de Crenças acerca das Doenças Mentais (ICDM)}

A versão breve do ICDM de Loureiro (2008) é constituída por 23 itens em formato de resposta tipo Likert de 1 (discordo completamente) a 6 pontos (concordo completamente) que se reportam às dimensões originais do instrumento, nomeadamente incurabilidade, reconhecimento da doença, doença como causa de estigma e descriminação, perigosidade, responsabilidade individual e doença como condição médica. Os estudos em que esta versão foi aplicada apresentam bons índices de consistência interna e uma estrutura fatorial em sintonia com a derivação teórica e conceptual na qual assentou a sua construção (Loureiro, 2008).

\section{Escala de Avaliação do Estigma Pessoal (EAEP)}

A versão portuguesa da EAEP (Loureiro \& Abrantes, 2014) é constituída por sete itens em formato de resposta Likert de 1 (discordo completamente) a 5 (concordo completamente) pontos. A resposta aos itens é precedida pela vinheta já referida no QuALiSMental. A escala foi submetida a uma Análise Fatorial Confirmatória (AFC) revelando uma estrutura com dois fatores, nomeadamente: fraco-não doente e perigosidade-imprevisibilidade. Esta escala apresenta boa qualidade psicométrica (Loureiro \& Abrantes, 2014).

\section{Procedimentos}

O projeto de intervenção foi previamente submetido à Comissão de Ética da Unidade de Investigação em Ciências da Saúde: Enfermagem (UICISA:E) da Escola Superior de Enfermagem de Coimbra (N.: P60306/2019), tendo obtido parecer positivo.

Foi também apresentada a proposta de realização da intervenção à diretora da instituição, tendo sido obtido parecer favorável e autorizada a sua aplicação, em consonância com o Conselho Pedagógico da instituição que incluiu o programa nas atividades da semana de integração ao curso. 
Todos os estudantes foram contactados e convidados pela coordenadora do primeiro ano do curso de licenciatura para participar voluntariamente no programa. Aquando do contacto, foram-lhe explicados os objetivos e natureza do programa, assim como todos os aspetos e questões relativas à sua participação. Os que manifestaram o seu interesse em participar foram divididos aleatoriamente por grupos e distribuídos aleatoriamente pela equipa de formadores do programa, sendo todos os formadores especialistas em saúde mental. Todos os participantes leram e assinaram o consentimento informado.

A intervenção, com a duração de nove horas, foi composta por quatro sessões, três de duas horas e uma de três horas, e decorreu em setembro de 2019.

A colheita de dados foi efetuada antes da intervenção e após a intervenção. Os conteúdos abordados no programa de PASM incluíram todas as componentes da LSM, destacando-se os conceitos de saúde e problemas de saúde mental (e perturbações mentais), reconhecimento de sinais e valorização de sintomas associados à depressão, fatores de risco associados, e especificamente as estratégias do plano de ação conducentes à intervenção em termos de primeira ajuda em saúde mental. No caso das situações de crise e que implicam primeiros socorros, foi dado enfase às situações que envolvem ideação suicida e respetivo plano de ação.

\section{Análise Estatística}

A análise foi efetuada com recurso ao programa IBM SPSS, versão 24. Foram calculadas as medidas estatísticas resumo adequadas, incluindo as distribuições absolutas e percentuais. Como testes de hipótese estatísticos, foram aplicados o teste de McNemar e o teste $t$ de Student para grupos emparelhados. As medidas de tamanho de efeito calculadas foram respetivamente $\circ g$ (teste de McNemar) e o $d$ ( $t$ de Student) de Cohen (1988). Para a interpretação dos resultados destas medidas, utilizaram-se como valores referência: pequeno se 0,05 $<g<0,15$; médio se 0,15 $\leq g<0,25$ e grande se $g \geq 0,25$. Em termos do $d$, foram considerados pequenos se $0,20 \leq d<0,50$; médios se $0,50 \leq d<0,80$ e grandes se $d \geq 0,80$.

Nos casos da aplicação do teste de McNemar, e dado tratarem-se de tabelas de $2 \times 2$, foram ainda determinadas e adicionadas as tabelas de resultados as percentagens correspondentes à concordância (CD), tenho como base o somatório dos casos nas células A e D da diagonal principal da matriz, divididas pelo tamanho da amostra em que $C D=(A+D) / n$.

No caso do ICDM e da EAEP, optou-se por realizar a análise estatística item a item, no sentido desta análise permitir escrutinar de forma mais abrangente os conteúdos e principais mensagens vinculadas e abordados com o programa. 


\section{Resultados}

Relativamente à componente de reconhecimento dos problemas e perturbações mentais de modo a promover e facilitar a procura de ajuda (Tabela 1), observou-se com a formação, um aumento com significado estatístico e tamanhos de efeito considerados grandes nas percentagens com que foram assinalados os rótulos "depressão" $(p<0,001 ; g=0,43)$, "doença mental" $\left(\chi^{2}=22,781 ; p<0,001 ; g=\right.$ $0,44)$.

Em sentido oposto, constatou-se também uma diminuição, após a intervenção, nas percentagens com que foram assinalados os rótulos "bulimia" ( $p=0,004 ; g=0,50)$, "stresse" $\left(\chi^{2}=21,951 ; p<0,001\right.$; $g=0,38)$, "esgotamento nervoso" $\left(\chi^{2}=17,633 ; p<0,001 ; g=0,40\right)$, "problemas psicológicos/mentais/emocionais" ( $\left.\chi^{2}=12,250 ; p<0,001 ; g=0,31\right)$ e "ansiedade" $\left(\chi^{2}=12,595 ; p<\right.$ $0,001 ; g=0,29)$.

As percentagens de concordância permitiram-nos verificar que as mudanças mais vincadas [usando como medida o valor obtido de 1-CD, isto é, percentagens nas células que indicam discordância ( $B$ e C)], ocorreram nos rótulos "stress", "ansiedade", "problemas psicológicos/mentais/emocionais" e "doença mental".

No que respeita à valorização das expressões de uso corrente no quotidiano, perspetivando-as como úteis ou prejudiciais, verificámos que previamente à intervenção, a utilização e expressões como "és superior a isso, eu conheço-te bem!" (90,0\%), "a seguir à tempestade há sempre a bonança!" (86,0\%), "estou preocupado contigo!" (66,0\%), "precisas colocar um sorriso nesses lábios!" (88,0\%) ou então "tens que ser forte! Tu consegues... é tudo "força de vontade" (79,0\%), foram perspetivadas como úteis pela grande maioria dos participantes. Em sentido oposto, as expressões "tens tudo, não tens motivo nenhum para andares assim!", "a forma como te tens comportado não é correta!" e "há tanta gente com problemas piores que os teus!" foram vistas como prejudiciais para dizer a alguém que está em sofrimento.

A comparação efetuada antes e depois da intervenção revelou mudanças como significado estatístico em $84,0 \%$ dos itens. Contudo o incremento na perceção de utilidade apenas se observou na frase "estou preocupado contigo!" $\left(\chi^{2}=21,441 ; p<0,001 ; g=0,41\right)$, que passou de $66,0 \%$ para $94,0 \%$. Nos restantes itens diminuiu a perceção de utilidade das frases, sendo essas mudanças associadas a tamanhos de efeito elevados, uma vez que todos os valores do $g$ foram $\geq 0,25$. 


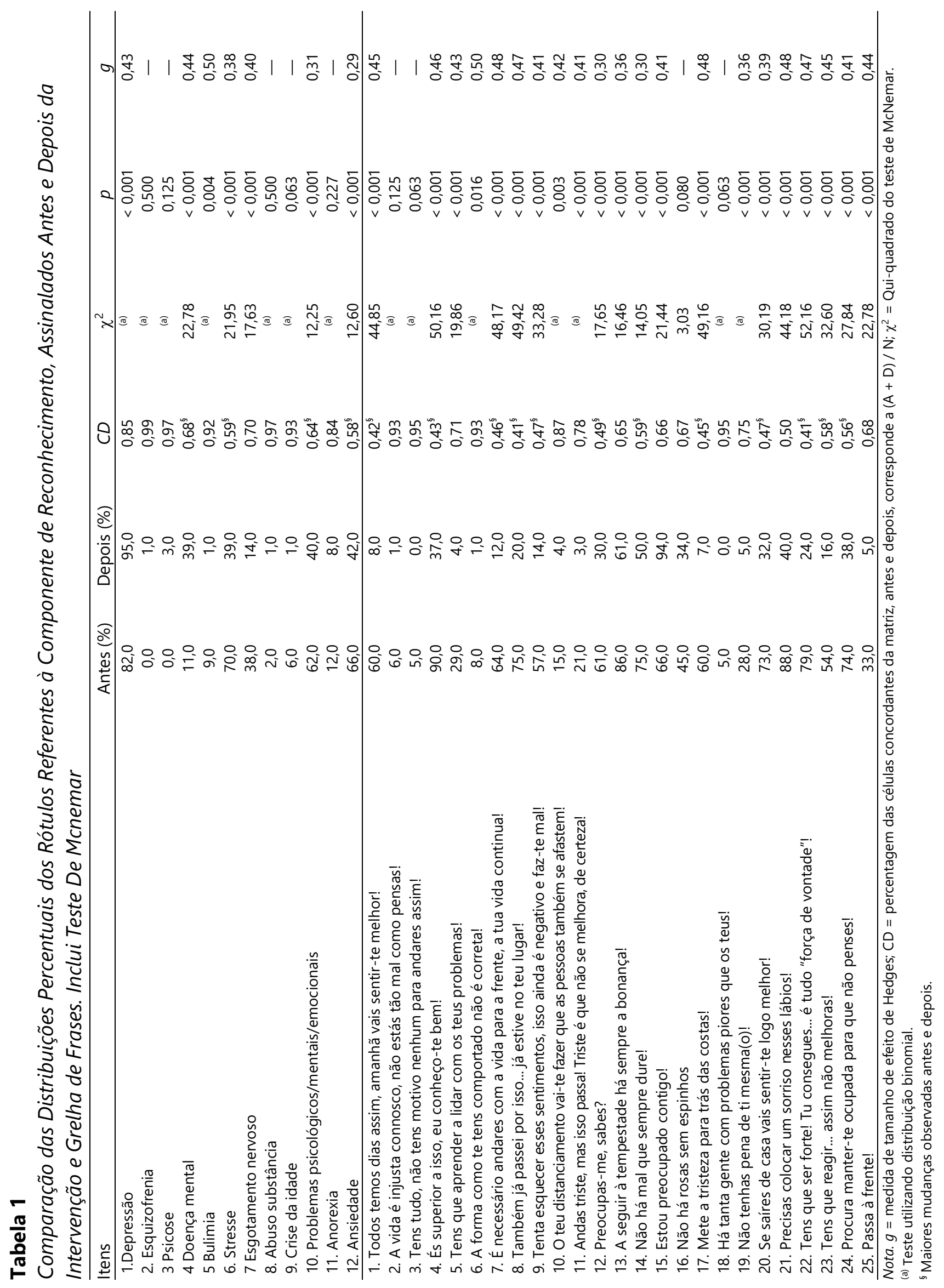


Relativamente às crenças e atitudes acerca das doenças e doentes mentais, a análise efetuada aos itens do ICDM, antes e depois da intervenção (Tabela 2), permitiram verificar mudanças com significado estatístico em $70,0 \%$ dos itens do inventário.

Como se pode observar a partir das médias obtidas (antes e depois), a concordância diminuiu nos itens "as doenças mentais são cíclicas (voltam de tempos a tempos)" ( $t=1,80 ; p=0,004 ; d=0,18$ ), "se as pessoas tiverem cuidado com elas próprias, podem evitar as doenças mentais" ( $t=4,69 ; p<0,001$; $d=$ $0,47)$, "as doenças mentais das pessoas resultam da falta de cuidados" ( $t=3,78 ; p<0,001 ; d=0,40)$, "o comportamento de uma pessoa com doença mental é imprevisível" ( $t=4,22 ; p<0,001$; $d=0,42)$, "As doenças mentais requerem mais tempo para serem curadas do que as outras doenças" $(t=1,77 ; p<$ $0,001 ; d=0,18)$, "a pessoa com doença mental é mais propensa a tornar-se criminosa" $(t=5,27 ; p<0,001$; $d=0,53)$, "se vier a sofrer de uma doença mental é porque não tive o cuidado que devia ter" $(t=3,55 ; p$ $<0,001 ; d=0,36)$, "os doentes mentais têm tendência a serem perigosos" ( $t=5,39 ; p<0,001 ; d=0,54)$; "tomar medicamentos ao longo da vida para as doenças mentais torna (...) dependentes...." $(t=2,27 ; p$ $=0,007 ; d=0,23)$, "os indivíduos que são diagnosticados como doentes mentais, terão os sintomas ..." $(t=2,52 ; p=0,007 ; d=0,25)$.

Nos itens referidos, as medidas de tamanho de efeito variaram de $d=0,18$ a $d=0,54$, sendo que apenas em dois itens foram elevados ("a pessoa com doença mental é mais propensa a tornar-se criminosa" e "os doentes mentais têm tendência a serem perigosos"), sendo pequenos nos restantes. Os itens das crenças em que se observou um acréscimo na concordância e cuja mudança se revestiu de significado estatístico foram "as pessoas com doença mental estão aptas para viver nas suas comunidades..." ( $t=-2,94 ; p=0,002 ; d=0,29)$; "os atrasos nos tratamentos pioram o sucesso de cura das doenças mentais" ( $t=-1,89 ; p=0,030 ; d=0,18$ ); "o tratamento inicial das doenças mentais requer o uso de medicamentos" ( $t=-3,23 ; p=0,001 ; d=0,32$ ); "posso vir a sofrer de uma doença mental independentemente do que fizer ou não para isso" ( $t=-5,22 ; p=, 000 ; d=0,52)$; "a reabilitação é eficaz no melhoramento das doenças mentais" ( $t=-2,52 ; p=, 007 ; d=0,20)$; "a medicação é eficaz na melhoria dos sintomas na doença mental" ( $t=-2,79 ; p=, 003 ; d=0,28)$. Neste caso, exceção para o item "posso vir a sofrer de uma doença mental independentemente do que fizer ou não para isso", em que se observou um tamanho de efeito elevado $(d=0,52)$, todos os outros indicaram tamanhos de efeito considerados pequenos.

Relativamente aos itens do estigma pessoal, verificámos que apesar da redução das médias em todos os itens serem estatisticamente significativas, os itens cujas mudanças foram maiores foram, "se a Sofia quisesse poderia sair desta situação por si" $(t=7,87 ; p<0,001 ; d=0,79)$ e "a situação da Sofia é um sinal de fraqueza pessoal" ( $t=5,44 ; p<0,001 ; d=0,54)$. 


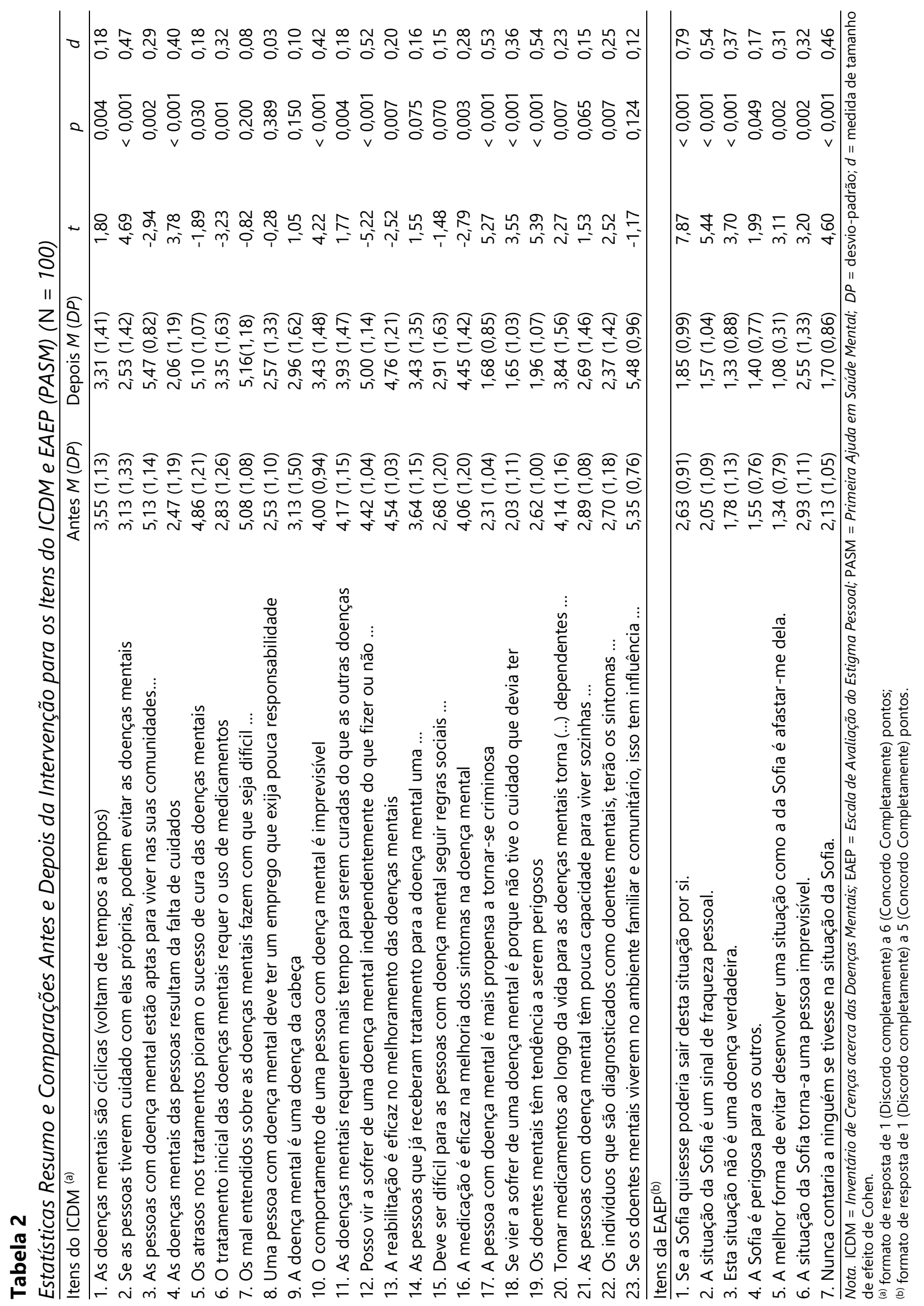




\section{Discussão}

Iniciamos a discussão pela referência às principais limitações e fragilidades deste tudo, sobretudo decorrentes do desenho do estudo utilizado (grupo único e avaliação pré e pós-intervenção). É de destacar a não existência de um grupo de controlo, o que constitui uma ameaça à validade, dado que não existe um termo de comparação que permita avaliar o efeito do programa comparativamente a outro tipo de intervenção concorrente, ou à ausência de uma intervenção. Ainda que o desenho comporte uma avaliação pré e pós intervenção, julgamos que a não existência de follow-up, é também ela uma limitação que não nos permite avaliar a estabilidade do conhecimento adquirido pelos jovens estudantes ao longo do tempo e neste sentido maximizar a análise da efetividade da intervenção.

É de salientar que os objetivos do programa são voltados para a ação em prol da saúde mental do indivíduo e daqueles que o rodeiam e neste sentido não se centram apenas no aumento do conhecimento e diminuição das crenças e atitudes estigmatizantes, mas pretende capacitar os participantes para agirem como prestadores de primeira ajuda em saúde mental, treinando por exemplo o algoritmo de ação com recurso a role-play e a análises e discussão de casos no sentido de promover a ajuda e incentivar a procura de ajuda em saúde mental. Salienta-se também que o programa não ensina ou serve para fazer diagnósticos em saúde mental, deixando claro nos conteúdos que as questões diagnósticas estão associadas a profissionais de saúde com formação especifica para esse efeito (e.g., psiquiatra) (Loureiro, 2014).

No que respeita aos resultados obtidos na componente de reconhecimento dos problemas (e perturbações mentais) de modo a promover e facilitar a procura de ajuda, os resultados são globalmente positivos, o que pode constatar-se pelo perfil das mudanças nas frequências com que os rótulos são assinalados. Essas mudanças, seja pelo aumento ou pela redução, configuram o reconhecimento dos sinais e valorização dos sintomas, e indiciam a capacidade dos jovens em problematizar a situação descrita na vinheta, atribuindo-Ihe um significado através do uso de um rótulo adequado (Loureiro, 2014). Neste sentido, é positivo verificar o incremento nos rótulos "depressão" e "doença mental", e o decréscimo no assinalar dos rótulos "bulimia", stress", "ansiedade", "problemas psicológicos/mentais/emocionais" e "esgotamento nervoso". Como se referiu, a intenção não é apelar em qualquer momento ao diagnóstico em saúde mental, mas, a partir de uma problematização adequada, potenciar a prestação de primeira ajuda.

Estes resultados estão consonância com os encontrados noutros estudos realizados no contexto Português (Cunha, 2019; Loureiro \& Costa, 2019; Loureiro \& Freitas, 2020; Loureiro \& Sousa, 2019) e mesmo internacionais (Bond et al., 2015; Burns et al., 2017), em que se observa um incremento dos rótulos ajustados para a descrição da situação evocada (depressão) e uma diminuição naqueles que não configuram nem se ajustam à situação. 
No que respeita às frases que são comumente utilizadas como forma de expressar preocupação, apoio e suporte à pessoa em situação de sofrimento psicológico (associado à depressão), podemos verificar que no cômputo geral as mudanças observadas também elas são positivas, em especial o incremento observado na adequação do uso da expressão "Estou preocupado contigo!" (Loureiro, 2014).

Em simultâneo observa-se uma diminuição da perceção de utilidade de frases que indiciam ou podem ser entendidas como uma forma de desvalorização ou mesmo acusação e consequente julgamento do que a pessoa está a sentir, podendo levar a pessoa a afastar-se e a recursar a ajuda. São exemplos, "és superior a isso, eu conheço-te bem!", "também já passei por isso... já estive no teu lugar!", "precisas colocar um sorriso nesses lábios!" e "tens que ser forte! Tu consegues... é tudo "força de vontade".

Tal como expressado por Bond et al. (2019), estas expressões e outras (e.g., "mete a tristeza para trás das costas!", "se saíres de casa vais sentir-te logo melhor!" ou "passa à frente!"), não se ajustam ao contexto, pois não expressam suporte e apoio; não configuram respeito pelo que a pessoa está a sentir; nem são formas de exprimir apoio emocional consistente e, por isso, não encorajam a pessoa a expressar os seus sentimentos e a procurar ajuda em saúde mental (Loureiro et al., 2014).

Estes resultados podem ser entendidos no contexto dos conteúdos que são privilegiados na intervenção, sobretudo em termos do ensino, treino e domínio do plano de ação para primeira ajuda em saúde mental, com destaque para as duas primeiras ações do plano ANIPI ("Aproximar-se da pessoa, observar e ajudar" e "Não julgar e escutar com atenção". A abordagem feita aos princípios de comunicação eficaz para escutar sem fazer juízos de valor, prestando especial atenção às atitudes (princípios de aceitação, honestidade e empatia) e o que elas transmitem, assim como as habilidades da comunicação (verbais e não verbais) (Loureiro, 2014) justificam em parte o decréscimo observado no sentido de utilidade destas expressões no quotidiano.

No que concerne às crenças e atitudes estigmatizantes acerca das doenças mentais, incluindo os resultados observados nos itens das duas escalas (ICDM e EAEP), podemos verificar que ambos são positivos e mostram que o programa de PASM pode ser utilizado como medida para combater as crenças e atitudes estigmatizantes associadas às doenças e doentes mentais. Por um lado, contribui para uma redução substancial nas médias dos itens que consubstanciam uma visão estigmatizante e estereotipada das doenças, sendo mais vincada para o caso da perigosidade ("a pessoa com doença mental é mais propensa a tornar-se criminosa", "os doentes mentais têm tendência a serem perigosos"), imprevisibilidade ("o comportamento de uma pessoa com doença mental é imprevisível") e a doença como sinal de fraqueza pessoal ("a situação da Sofia é um sinal de fraqueza pessoal").

Os três estereótipos referidos são os que surgem com expressão mais vincados na literatura (Loureiro, 2008; Loureiro et al., 2008), sendo também referidos como entraves à procura de ajuda em saúde mental. Estes resultados estão em consonância com as análises efetuadas em revisões sistemáticas com metaanálise acerca do efeito do programa no estigma social associados as doenças e doentes mentais (Morgan et al., 2018). 
Observa-se também um aumento substancial nas médias dos itens que configuram uma visão realista das doenças, assente por exemplo na reabilitação psicossocial como intervenção conducente à melhoria da situação de saúde das pessoas com doenças mentais diagnosticadas, os psicofármacos como coadjuvante nos tratamentos e, não menos importante, a responsabilidade individual pela manutenção da saúde mental, no sentido de apelar para estilos de vida saudáveis.

Estes resultados, em linha com outros estudos em que é aplicada a mesma intervenção (Loureiro \& Costa, 2019; Loureiro \& Freitas, 2020) mostram resultados comparativamente mais positivos na mudança do estigma pessoal, quando comparados com intervenções em que é privilegiado, por exemplo, o contacto com pessoas com doença mental diagnosticada no âmbito dos ensinos clínicos dos estudantes de Enfermagem (Gil et al., 2016).

Alguns itens das crenças apresentam resultados opostos ao que seria o expectável com a frequência do programa, nomeadamente nos itens, "se as pessoas tiverem cuidado com elas próprias, podem evitar as doenças mentais" e "posso vir a sofrer de uma doença mental independentemente do que fizer ou não para isso". Estes resultados, podem dever-se ao entendimento que os participantes fazem de algumas mensagens veiculadas no programa, nomeadamente a "universalidade e democratização" dos problemas e perturbações mentais como uma realidade que pode afetar qualquer um em qualquer momento das suas vidas (Loureiro, 2008).

Em termos globais com a intervenção podemos verificar um outro dado e que decorre do próprio conceito de literacia em saúde mental, nomeadamente porque envolve a constelação de crenças e conhecimentos acerca dos problemas e perturbações mentais que ajudam no seu reconhecimento, gestão e prevenção (Jorm, 2019). Se observarmos os resultados das medidas de tamanho de efeito calculadas no computo das componentes sob estudo, podemos verificar que os valores são mais elevados nas componentes relativas aos conhecimentos, comparativamente às crenças e atitudes, ainda que em ambos os casos com significado estatístico. Este dado está em consonância com aquele que é o reportório teórico e empírico que aponta para a dificuldade em mudar crenças enraizadas social e culturalmente, e inclusive comportamentos relacionados com a saúde mental (Loureiro et al., 2008).

\section{Conclusão}

Os resultados deste estudo permitem concluir que programa de PASM é uma ferramenta psicoeducacional que pode ser utilizada como medida conducente ao incremento da LSM acerca da depressão. Promove o conhecimento em saúde mental ao nível do reconhecimento e valorização dos problemas e perturbações mentais e capacita os estudantes para a prestação de primeira ajuda em saúde mental interpares, sendo por isso voltada para a ação em prol da saúde mental. Simultaneamente permite reduzir as crenças e atitudes estigmatizantes e deste pode potenciar a procura de ajuda em saúde mental. O plano de ação (ANIPI), especificamente nas estratégias de "Aproximar-se da pessoa, observar e ajudar" e "Não julgar e escutar com atenção" são momentos fundamentais na prestação de primeira ajuda e 
apoio. A utilização de expressões adequadas e baseadas em atitudes comunicacionais eficazes são peças fundamentais que favorecem a procura de uma solução para os problemas e a recuperação da boa saúde mental. Neste sentido o programa promove a consciencialização de que a procura de ajuda em saúde mental é uma resposta adequada e possível nas situações que envolvem sofrimento e consequente adoecer mental.

\section{Conflito de interesses | Conflict of interest: nenhum | none.}

Fontes de financiamento | Funding sources: nenhuma | none.

Contributos: LL: Desenho do estudo; Pesquisa bibliográfica; Revisão da literatura; Preparação das medidas e escrita do protocolo; Recrutamento dos participantes; Análise estatística; Escrita do manuscrito; Supervisão da escrita do manuscrito e aprovação do manuscrito final. AR: Desenho do estudo; Pesquisa bibliográfica; Revisão da literatura; Preparação das medidas e escrita do protocolo; Recrutamento dos participantes; Análise estatística; Escrita do manuscrito; Supervisão da escrita do manuscrito e aprovação do manuscrito final. CP: Desenho do estudo; Pesquisa bibliográfica; Revisão da literatura; Preparação das medidas e escrita do protocolo; Recrutamento dos participantes; Análise estatística; Escrita do manuscrito; Supervisão da escrita do manuscrito e aprovação do manuscrito final. SC: Desenho do estudo; Pesquisa bibliográfica; Revisão da literatura; Preparação das medidas e escrita do protocolo; Recrutamento dos participantes; Análise estatística; Escrita do manuscrito; Supervisão da escrita do manuscrito e aprovação do manuscrito final. LC: Pesquisa bibliográfica; Revisão da literatura; Preparação das medidas e escrita do protocolo; Recrutamento dos participantes; Análise estatística; Escrita do manuscrito, revisão final e aprovação do manuscrito. SCorreia: Pesquisa bibliográfica; Revisão da literatura; Preparação das medidas e escrita do protocolo; Recrutamento dos participantes; Análise estatística; Escrita do manuscrito, revisão final e aprovação do manuscrito. TM: Análise estatística; Revisão final e aprovação do manuscrito.

\section{Referências}

Associação Americana de Psiquiatria. (2014). DSM-5: Manual de diagnóstico e estatística das perturbações (5a ed.). Artmed Editora.

Bond, K. S., Jorm, A. F., Kitchener, B. A., \& Reavley, N. J. (2015). Mental health first aid training for Australian medical and nursing students: An evaluation study. BMC Psychology, 3, Artigo 11. http://doi: 10.1186/ s40359-015-0069-0

Bond, K.S., Cottrill, F.A., Blee, F.L., Kelly, C.M., Kitchener, B. A., \& Jorm, A. F. (2019). Offering mental health first aid to a person with depression: A Delphi study to re-develop the guidelines published in 2008. $B M C$ Psychology, 7, Artigo 37. https://doi.org/10.1186/s40359-019-0310-3

Burns, S., Crawford, G., Hallett, J., Hunt, K., Chih, H. J., \& Tilley, P. J. M. (2017). What's wrong with John? A randomised controlled trial of Mental Health First Aid (MHFA) training with nursing students. BMC Psychiatry, 17(1), Artigo 111. https://doi.org/10.1186/s12888-017-1278-2

Cohen, J. (1988). Statistical power analysis for the behavioral sciences. Lawrence Erlbaum Associates.

Cunha, S. R. (2019). Efetividade do programa de primeiros socorros em saúde mental em estudantes do $1 .^{\circ}$ ano de Enfermagem [Dissertação de mestrado, Escola Superior de Enfermagem de Coimbra]. Repositório Científico da Escola Superior de Enfermagem de Coimbra. http://web.esenfc.pt/?url=H4DEplcb

Gil, I. M., Santos, J. C., \& Loureiro, L. M. J. (2016). Estigma em estudantes de enfermagem: antes e depois do contacto com pessoas com transtornos mentais. Revista Enfermagem UERJ, 24(1), Artigo e12309. https://doi.org/10.12957/reuerj.2016.12309 
Jorm, A. (2019). The concept of mental health literacy. Em O. Bauer, U. Levin-Zamir, D. P. Pinheiro, \& K. Sorensen (Eds.), International handbook of health literacy: Research, practice and policy across the life-span (pp. 5366). Policy Press.

Jorm, A. F., Korten, A. E., Jacomb, P. A., Christensen, H., Rodgers, B., \& Pollitt, P. (1997). "Mental health literacy": A survey of the public's ability to recognize mental disorders and their beliefs about the effectiveness of treatment. Medical Journal of Australia, 166(4), 182-186. https://doi.org/10.5694/j.13265377.1997.tb140071.x

Kitchener, B. A., \& Jorm, A. F. (2002). Mental health first aid training for the public: Evaluation of effects on knowledge, attitudes and helping behavior. BMC Psychiatry, 2(1), Artigo 10. https://doi.org/10.1186/1471$244 \mathrm{X}-2-10$

Loureiro, L. (2014). Primeira ajuda em saúde mental. Escola Superior de Enfermagem de Coimbra.

Loureiro, L. M. \& Freitas, P. M. (2020). Effectiveness of the mental health first aid program in undergraduate nursing students. Revista de Enfermagem Referência, 5(1), Artigo e19078. https://doi.org/10.12707/RIV19078

Loureiro, L. M. J. (2008). Representações sociais da loucura: Importância para a promoção da saúde mental. [Tese de doutoramento não publicada], Universidade do Porto.

Loureiro, L. M. J. (2015). Questionário de Avaliação da Literacia em Saúde Mental - QuALiSMental: Estudo das propriedades psicométricas. Revista de Enfermagem Referência, 4(4), 79-88. https://doi.org/10.12707/RIV14031

Loureiro, L. M. J. (2020). Avaliação de impacto do programa de Primeira Ajuda em Saúde Mental na literacia em saúde mental dos estudantes do $1 .^{\circ}$ ano do curso de licenciatura em Enfermagem [Relatório de pósdoutoramento não publicado]. Universidade do Porto.

Loureiro, L. M. J., Dias, C. A., \& Oliveira, R. A. (2008). Crenças e Atitudes acerca das doenças e dos doentes mentais. Contributos para o estudo das representações sociais da loucura. Referencia, (8). 33-44. Recuperado de http://www.index-f.com/referencia/2008pdf/8-3344.pdf

Loureiro, L. M. J., Mendes, A. M. O. C., Barroso, T. M. M. D. A., Santos, J. C. P., Oliveira, R. A., \& Ferreira, R. O. (2012). Literacia em saúde mental de adolescentes e jovens: Conceitos e desafios. Revista de Enfermagem Referência, 3(6), 157-166. https://doi.org/10.12707/RIII11112

Loureiro, L. M. J., Sousa, C. S. F., \& Gomes, S. P. S. (2014). Primeira ajuda em saúde mental — Pressupostos e linhas orientadoras de ação. Em L. M. J. Loureiro (Coord.), Literacia em saúde mental: Capacitar as pessoas e as comunidades para agir (Vol. 8, pp. 63-77). Unidade de Investigação em Ciências da Saúde: Enfermagem (UICISA:E).

Loureiro, L. M., \& Sousa, C. F. (2019). Programa de Primeiros Socorros em Saúde Mental: Estudo piloto. Revista Portuguesa de Investigação Comportamental e Social, 5(1), 72-86. https://doi.org/10.31211/rpics.2019.5.1.108

Loureiro, L., \& Costa, L. (2019). Avaliação do programa de primeiros socorros em saúde mental em estudantes de licenciatura em enfermagem. Revista de Enfermagem Referência, 4(20), 9-18. https://doi.org/10.12707/RIV18087

Loureiro, L., \& Sousa, C. (2019). Programa de primeiros socorros em saúde mental: Estudo piloto. Revista Portuguesa de Investigação Comportamental e Social, 5(1), 72-86. https://doi.org/10.31211/rpics.2019.5.1.108

Loureiro, L., \& Abrantes, A. (2014). Propriedades psicométricas das Escalas de Avaliação do Estigma associado ao abuso de álcool. Interações: Sociedade e as Novas Modernidades, 27, 47-59. https://www.interacoesismt.com/index.php/revista/article/view/379

Morgan, A. J., Ross, A., \& Reavley, N. J. (2018). Systematic review and meta-analysis of mental health first aid training: Effects on knowledge, stigma, and helping behaviour. PLOS ONE, 13(5), Artigo e0197102. https://doi.org/10.1371/journal.pone.0197102 